\title{
Avaliação através da tomografia por coerência óptica do esmalte dentário após o uso de dentifrícios clareadores
}

\author{
Evaluation through tomography by optical coherence of dental enamel after \\ the use of clearing dentifrices

\section{Bárbara Andrade Leimig RODRIGUES ${ }^{a}$ (D), Letícia Santos Alves de MELOa* (D), Rafael Antônio de Oliveira RIBEIRO ${ }^{b}$ (1) , Alexandre Batista Lopes do NASCIMENTOa ${ }^{(0)}$, Hilcia Mezzalira TEIXEIRAa ${ }^{a}$} \\ aUFPE - Universidade Federal de Pernambuco, Centro de Ciências da Saúde, Departamento de Prótese e Cirurgia Buco \\ Facial, Recife, PE, Brasil \\ bUNESP - Universidade Estadual Paulista, Faculdade de Odontologia de Araraquara, Departamento de Materiais \\ Odontológicos e Prótese, Araraquara, SP, Brasil
}

\begin{abstract}
Como citar: Rodrigues BAL, Melo LSA, Ribeiro RAO, Nascimento ABL, Teixeira HM. Avaliação através da tomografia por coerência óptica do esmalte dentário após o uso de dentifrícios clareadores. Rev Odontol UNESP. 2019;48:e20190078. https://doi.org/10.1590/1807-2577.07819
\end{abstract}

\begin{abstract}
Resumo
Introdução: Historicamente, materiais abrasivos, como mármore em pó, corais e cinzas ósseas, eram utilizados para higienização dentária. Com a evolução, tais materiais foram substituídos por dentifrícios aplicados em escovas dentais, com a mesma finalidade. Objetivo: Avaliar, através da Tomografia por Coerência Óptica, o desgaste do esmalte dentário, após o uso de diferentes escovas dentais e materiais utilizados na escovação com componentes abrasivos. Material e método: Foram confeccionados 50 corpos de prova, distribuídos em 10 grupos ( $\mathrm{n}=5$ ), de acordo com o dentifrício/escova utilizado. Para o grupo A, foi utilizada a escova dental Curaprox ${ }^{\circledR}$ Adulto Ultra Macia, e, para o grupo B, a escova Dental $\mathrm{K}^{\circledR}$ - escova adulto macia. Foram utilizados os seguintes materiais para escovação: Curaprox ${ }^{\circledR}$ Black is White; Colgate ${ }^{\circledR}$ Luminous White Instant, Close Up ${ }^{\circledR}$ White Attraction - MenSuperpure; Carvão Ativado; Água Destilada. As imagens foram realizadas antes e depois da ciclagem de escovação e comparadas quanto às alterações de superfície. A perda da estrutura superficial foi avaliada através dos métodos qualitativo e quantitativo. Para análise estatística dos dados obtidos, foram realizadas as medidas estatísticas: média, desvio padrão, mediana e percentis, avaliados inferencialmente através dos testes estatísticos Mann-Whitney, Kruskal-Wallis, com nível de significância de 5\%. Resultado: Maior índice de perda de estrutura superficial do esmalte nos grupos G2A $(16,09)$ e G2B $(11,38)$ e menor índice de perda estrutural nos grupos G5A $(1,07)$ e G5B $(1,20)$. Conclusão: Através desse estudo, observou-se que os dentifrícios clareadores e o Carvão Ativado são capazes de promover intenso desgaste do esmalte dentário.
\end{abstract}

Descritores: Dentifrícios; esmalte dentário; abrasão dentária; materiais dentários.

\begin{abstract}
Introduction: Historically, abrasive materials such as marble powders, corals and bone ashes were used for dental hygiene. With evolution, these materials were replaced by dentifrices applied on toothbrushes for the same purpose. Objective: To evaluate, through Optical Coherence Tomography, the wear of dental enamel after the use of different toothbrushes and materials used in brushing with abrasive components. Material and method: 50 specimens were prepared, distributed in 10 groups $(n=5)$ according to the toothpaste used. For group A, the Curaprox ${ }^{\circledR}$ Adult Ultra Macia toothbrush was used and for group B, the soft toothbrush $\mathrm{K}^{\circledR}$ - soft adult toothbrush. The following brushing materials were used: Curaprox ${ }^{\circledR}$ Black is White; Colgate ${ }^{\circledR}$ Luminous White Instant, Close Up ${ }^{\circledR}$ White Attraction - MenSuperpure; Activated Charcoal; Distilled Water. The images were performed before and after the brushing cycle and were compared for surface changes. The loss of the surface structure was evaluated through qualitative and quantitative methods. Statistical analysis of the data was performed using the statistical methods Mann-Whitney,
\end{abstract}


Kruskal-Wallis, with significance level of 5\%, mean, standard deviation, median and percentiles. Result: A higher index of loss of enamel structure was observed in the G2A (16.09) and G2B (11.38) groups and a lower structural loss rate in the specimens corresponding to the groups G5A $(1,07)$ and G5B $(1,20)$. Conclusion: Through this study it was observed that the whitening dentifrices and the Activated Charcoal are able to promote an intense wear of the dental enamel.

Descriptors: Dentifrices; dental enamel; tooth abrasion; dental materials.

\section{INTRODUÇÃO}

Historicamente, a beleza sempre foi uma das grandes preocupações do ser humano, bem como a necessidade de limpeza dentária. Materiais abrasivos como mármore em pó, conchas, corais e cinzas ósseas, eram utilizados para remoção mecânica de detritos. Com a evolução, tais materiais foram substituídos pela aplicação de dentifrícios em escovas de dentes, como método primário para os mesmos fins ${ }^{1}$.

Segundo Rosenthaler, Randel ${ }^{2}$, um sorriso branco é tido como padrão de beleza jovem e harmônico. Dentes claros e bem alinhados representam aspectos importantes para um sorriso considerado perfeito pelos padrões de estética facial e dental atualmente aceitos pela sociedade, sendo sinônimo de saúde e beleza ${ }^{3}$. Dentes desalinhados e com colorações indesejáveis influenciam a autoestima e a auto aceitação do paciente, podendo repercutir negativamente nas relações interpessoais e profissionais ${ }^{3}$.

A mídia exerce grande influência psicológica sobre o padrão de sorriso que deve ser esteticamente aceito pela sociedade, influenciando desde a escala de cor até o alinhamento dentário. A veiculação indiscriminada de propagandas de cosméticos odontológicos que prometem efeito clareador rápido constitui um perigo para a integridade dentária dos pacientes, tendo em vista que, na maioria das vezes, não há um embasamento científico que comprove os benefícios do produto e ausência de malefícios para o paciente.

As técnicas de clareamento dental, devido sua simplicidade e eficácia, têm sido cada vez mais valorizadas pelos pacientes como tratamento para alterações de cor na superfície dentária. No entanto, antes de qualquer um dos produtos de branqueamento disponíveis no mercado poder ser utilizado, é necessário primeiro olhar para a etiologia da descoloração dentária 4 .

Com o intuito de satisfazer a necessidade dos consumidores, o mercado começou a oferecer dentifrícios cada vez mais especializados, com características terapêuticas e cosméticas que prometem efeito clareador rápido e efetivo por meio de simples ação mecânica, em um curto período de tempo ${ }^{3,5}$.

No entanto, ao verificar a composição destes cremes dentais, pode-se relatar que não possuem quaisquer das substâncias responsáveis pela liberação de oxigênio e consequente ação clareadora, pois não possuem peróxidos em sua composição. As principais funções terapêuticas e cosméticas de um dentifrício clareador baseiam-se na remoção da placa bacteriana e do biofilme, prevenindo a cárie, removendo manchas extrínsecas, polindo o esmalte dentário, por consequência, prevenindo o acúmulo de placa e deposição de novos pigmentos superficiais através da ação dos agentes abrasivos ${ }^{1,3,6,7}$.

A incorporação do Carvão Ativado nos métodos de higiene oral foi registrada inicialmente por Hipócrates, na Grécia. É uma substância que apresenta excelentes propriedades adsorventes, com grau elevado de porosidade e ampla área de superfície entre as partículas ${ }^{8 \dagger}$.

Idealmente, as partículas abrasivas dos cremes dentais deveriam ser mais macias que o esmalte e a dentina, e mais duras que a mancha, para causar danos mínimos. 0 poder abrasivo das referidas substâncias é diretamente influenciado pela forma, tamanho, dureza, clivagem,

\footnotetext{
† Schimidt CG. Desenvolvimento de filtros de carvão ativado para remoção do cloro da água potável. Porto Alegre: Universidade Federal do Rio Grande do Sul; 2011.
} 
friabilidade, concentração, distribuição e regularidade das partículas e pH do dentifrício, bem como pelo tipo de escova, pela técnica e pela carga aplicada durante a escovação. Também devem possuir compatibilidade com outros componentes empregados na formulação, em especial o flúor, apresentar propriedades de viscosidade e vazão aceitáveis, sem comprometer aparência e sabor do produto 7,9 .

O objetivo deste trabalho foi avaliar, através da Tomografia por Coerência Óptica (OCT), o desgaste do esmalte dentário, após o uso de diferentes escovas dentais e materiais utilizados na escovação com componentes abrasivos.

\section{MATERIAL E MÉTODO}

Este trabalho consistiu em um estudo experimental laboratorial, que foi aprovado pelo Comitê de Ética da Universidade Federal de Pernambuco (UFPE) (76067417.5.0000.5208). A pesquisa foi desenvolvida no Núcleo de Pesquisa Clínica em Biomateriais da UFPE, no Laboratório de Fotônica e Biofotônica do Departamento de Física da UFPE, e no Laboratório de Biomecânica da FOP/UPE.

Foram obtidos 50 corpos de prova, através de 20 molares humanos, hígidos, extraídos por indicações cirúrgicas ou ortodônticas, obtidos do Banco de Dentes da Faculdade de Odontologia da UFPE. Os resíduos teciduais foram removidos através de profilaxia com pasta de pedra-pomes e água, e os dentes foram lavados em água corrente e armazenados em solução de clorexidina a $2 \%$ por $12 \mathrm{~h}$, para desinfecção. Após isso, foram armazenados em solução fisiológica a $0,9 \%$, sob refrigeração, por, no máximo, seis meses, como preconiza a ISO TR-1140510.

Os corpos de prova foram distribuídos em 10 grupos ( $\mathrm{n}=5$ ), de acordo com o material utilizado na escovação e a escova dental, conforme a descrição no Quadro 1.

Quadro 1. Grupos analisados na pesquisa, material utilizado na escovação, escovas dentais utilizadas e composição dos materiais

\begin{tabular}{|c|c|c|c|c|}
\hline GRUPOS & ABREVIAÇÃo & $\begin{array}{c}\text { MATERIAL } \\
\text { UTILIZADO NA } \\
\text { ESCOVAÇÃO } \\
\end{array}$ & $\begin{array}{l}\text { ESCOVA } \\
\text { DENTAL }\end{array}$ & COMPOSIÇÃO \\
\hline Grupo $1 \mathrm{~A}$ & G1 A & $\begin{array}{c}\text { Curaprox }^{\circledR} \text { Black } \\
\text { is White }\end{array}$ & $\begin{array}{c}\text { Curaprox }^{\circledR} \\
\text { Adulto Ultra } \\
\text { Macia }\end{array}$ & $\begin{array}{l}\text { Água, sorbitol, sílica hidratada, } \\
\text { glicerina, carbono, aroma, } \\
\text { bentonite, monofluorofosfato, Decil } \\
\text { glicosídeo, vitamina E, Goma } \\
\text { Xantana, Mica, Hidroxiapatita, } \\
\text { Acesulfame-K, Maltodextrina, } \\
\text { Dióxido de titânio, Benzoato de }\end{array}$ \\
\hline Grupo 1 B & G1 B & $\begin{array}{c}\text { Curaprox }{ }^{\circledR} \text { Black } \\
\text { is White }\end{array}$ & $\begin{array}{c}\text { Dental } \mathrm{K}^{\circledR} \text { - } \\
\text { escova adulto } \\
\text { macia }\end{array}$ & $\begin{array}{l}\text { sódio, Cloreto de potassium, Citrus } \\
\text { Limon Peel Oil, Lactato de metil, } \\
\text { Propionamida de Di-isopropilo de } \\
\text { Metil, Ethyl Menthane Carboxamide, } \\
\text { Hidróxido de sódio, Ácido citrico, } \\
\text { Lactoperoxidase, Glicose Oxidase, } \\
\text { Amyloglucosidase, Potassium } \\
\text { Thiocyanate, Sucrose, Zea Mays } \\
\text { Startch, Ceteryl Alcohol, Tin Oxide. }\end{array}$ \\
\hline Grupo $2 \mathrm{~A}$ & G2 A & $\begin{array}{c}\text { Colgate }^{\circledR} \\
\text { Luminous White } \\
\text { Instant }\end{array}$ & $\begin{array}{c}\text { Curaprox }^{\circledR} \\
\text { Adulto Ultra } \\
\text { Macia }\end{array}$ & $\begin{array}{l}\text { Água, silica hidratada, sorbitol, } \\
\text { glicerina, PEG-12, trifosfato de } \\
\text { pentassódio, pirofosfato de } \\
\text { tetrapotássio, laurilsulfato de sódio, }\end{array}$ \\
\hline
\end{tabular}


Quadro 1. Continuação...

\begin{tabular}{|c|c|c|c|c|}
\hline GRUPOS & ABREVIAÇÃo & $\begin{array}{l}\text { MATERIAL } \\
\text { UTILIZADO NA } \\
\text { ESCOVAÇÃOO }\end{array}$ & $\begin{array}{l}\text { ESCOVA } \\
\text { DENTAL }\end{array}$ & COMPOSIÇÃO \\
\hline Grupo 2 B & G2 B & $\begin{array}{c}\text { Colgate }^{\circledR} \\
\text { Luminous White } \\
\text { Instant }\end{array}$ & $\begin{array}{c}\text { Dental } \mathrm{K}^{\circledR} \text { - } \\
\text { escova adulto } \\
\text { macia }\end{array}$ & $\begin{array}{l}\text { aroma, goma de celulose, } \\
\text { cocamidopropil betaína, sacarina } \\
\text { sódica, goma de xantana, fluoruro de } \\
\text { sódio, hidróxido de sódio, } \\
\text { hidroxipropil metilcelulosa, } \\
\text { propilenglicol, polissorbato } 80 \text {, mica, } \\
\text { CI 74160, CI 77891, Dióxido de } \\
\text { titânio, CI73360, CI 17200, CI } 42051 \text {, } \\
\text { eugenol, fluoreto de sódio, } 1100 \text { ppm } \\
\text { de íon flúor. }\end{array}$ \\
\hline Grupo $3 \mathrm{~A}$ & G3 A & $\begin{array}{c}\text { Close Up }{ }^{\circledR} \text { White } \\
\text { Attraction - } \\
\text { MenSuperpure }\end{array}$ & $\begin{array}{c}\text { Curaprox }^{\circledR} \\
\text { Adulto Ultra } \\
\text { Macia }\end{array}$ & \multirow{2}{*}{$\begin{array}{l}\text { Sorbitol, Água, Silica Hidratada, Lariu } \\
\text { Sulfato, PEG-32, Aroma de goma de } \\
\text { celulose, Fosfato trissódio, Sacarina } \\
\text { de sódio, Fluoreto de sódio, PVM/MA } \\
\text { compolyme, MICA, CI 74160, CI } \\
\text { 42090, Dióxido de titânio, } 1.450 \text { ppm } \\
\text { íon flúor. }\end{array}$} \\
\hline Grupo 3 B & G3 B & $\begin{array}{c}\text { Close Up }^{\circledR} \text { White } \\
\text { Attraction - } \\
\text { MenSuperpure }\end{array}$ & $\begin{array}{c}\text { Dental } \mathrm{K}^{\circledR} \text { - } \\
\text { escova adulto } \\
\text { macia }\end{array}$ & \\
\hline Grupo 4 A & G4 A & $\begin{array}{l}\text { Carvão Ativado } \\
\text { (P.A.) }\end{array}$ & $\begin{array}{c}\text { Curaprox }^{\circledR} \\
\text { Adulto Ultra } \\
\text { Macia }\end{array}$ & \multirow{2}{*}{$\begin{array}{l}\text { Cloreto, Sulfato, Ferro, Metais } \\
\text { Pesados e Solúveis em água. }\end{array}$} \\
\hline Grupo 4 B & G4 B & $\begin{array}{l}\text { Carvão Ativado } \\
\text { (P.A.) }\end{array}$ & $\begin{array}{c}\text { Dental } \mathrm{K}^{\circledR} \text { - } \\
\text { escova adulto } \\
\text { macia }\end{array}$ & \\
\hline Grupo $5 \mathrm{~A}$ & G5 A & Água Destilada & $\begin{array}{c}\text { Curaprox }^{\circledR} \\
\text { Adulto Ultra } \\
\text { Macia }\end{array}$ & \multirow{2}{*}{ Água destilada } \\
\hline Grupo 5 B & G5 B & Água Destilada & $\begin{array}{c}\text { Dental } \mathrm{K}^{\circledR} \text { - } \\
\text { escova adulto } \\
\text { macia }\end{array}$ & \\
\hline
\end{tabular}

Os dentes foram seccionados nas superfícies vestibular e lingual nos sentidos mesiodistal e oclusocervical, com disco diamantado dupla-face (KG Sorensen ${ }^{\circledR}$ - Cotia, São Paulo, Brasil), sob abundante refrigeração, para obtenção de fragmentos medindo $5 \mathrm{~mm} \times 5 \mathrm{~mm}$. m seguida, estes fragmentos foram incluídos em resina acrílica quimicamente ativada (JET, Clássico Artigos Odontológicos ${ }^{\circledR}$ - São Paulo, São Paulo, Brasil). Para tal, foi confeccionada uma matriz com silicone de condensação (COLTENE ${ }^{\circledR}$ - Rio de Janeiro, Rio de Janeiro, Brasil) contendo um orifício central de $17 \mathrm{~mm}$ de diâmetro. Uma fita dupla face foi colocada em uma placa de vidro e o fragmento dental foi posicionado e levado à matriz de silicone, que estava coberta por resina acrílica. Dessa forma, foi possível obter uma superfície plana do corpo de prova. Após a polimerização da resina, as superfícies vestibulares foram polidas à mão utilizando-se lixas de papel abrasivo de óxido de alumínio de granulações 600, 1.000 e 1.200, refrigeração com água, para obtenção de uma superfície plana de esmalte.

Os corpos de prova foram analisados através da Tomografia por Coerência Óptica (OCT, sigla em inglês para Optical Coherence Tomography), disponível no Laboratório de Fotônica e Biofotônica do Departamento de Física da UFPE, antes e após a escovação. Foram previamente marcados em duas regiões da resina acrílica com auxílio de ponta diamantada esférica 1012 (KG Sorensen ${ }^{\circledR}$ ), para que pudessem ser colocados sempre na mesma posição na OCT, seguindo uma marcação previamente realizada com fita adesiva no equipamento. Dessa forma, 
foi possível comparar as possíveis alterações na superfície dos espécimes decorrentes do ensaio de escovação mecânica.

Antes de cada ciclo de escovação, todos os espécimes foram submetidos à ação de saliva artificial Saliform (Fórmula \& Ação ${ }^{\circledR}$ - São Paulo, São Paulo, Brasil) com pH 5,5, durante cinco minutos, para simular o "desafio ácido" que ocorre após uma refeição11. 0 ensaio de escovação mecânica foi realizado na máquina de escovação MSET (Elquip ${ }^{\circledR}$ - São Carlos, São Paulo, Brasil), localizada no Laboratório de Biomecânica da FOP/UPE.

Escovas dentais Curaprox ${ }^{\circledR}$ Adulto Ultra Macia (São Caetano do Sul, São Paulo, Brasil), utilizadas nos grupos G1A, G2A, G3A, G4A e G5A, e Dental $\mathrm{K}^{\circledR}$ - escova adulto macia (Taboão da Serra, São Paulo, Brasil), utilizada nos grupos G1B, G2B, G3B, G4B e G5B, foram fixadas à máquina de escovação e mantidas paralelamente aos corpos de prova; utilizou-se uma solução dos dentifrícios para a escovação (com exceção dos grupos G4A e G4B, e G5A e G5B, que foram escovados somente com Carvão Ativado (P.A.) e água destilada, respectivamente). As soluções foram obtidas através da mistura dos materiais utilizados para escovação com água destilada na proporção de 1:2, conforme a norma $\mathrm{ISO}^{12}$, preparados antes da utilização ${ }^{13}$. A escovação simulada foi efetuada com movimentos lineares, sob carga axial estática de $200 \mathrm{~g}$ e velocidade de 4,5 ciclos por segundo, entendendo-se como ciclo o movimento anteroposterior da escova dental $^{9}$. Cada grupo foi submetido a 30.000 ciclos de escovação, simulando o tempo total de três anos de escovação ${ }^{14}$.

Para análise quantitativa, foi selecionado um par de imagens (antes e após a escovação) para cada espécime e sobrepostas, quanto ao nivelamento, no programa de edição de imagem Adobe ${ }^{\circledR}$ Photoshop, e a medição foi realizada no programa Image J (versão bundled para Mac), para verificar se houve ou não desgaste da superfície. Foram realizadas 11 medições de cada imagem selecionada. A calibração foi feita em Pixels (Px), 1062Px na linha horizontal (Eixo x) e 350Px na linha vertical (Eixo y). Os dados obtidos foram agrupados em um banco de dados digitados na planilha no Microsoft ${ }^{\circledR}$ EXCEL e, em seguida, analisados estatisticamente.

Os dados foram expressos através das medidas estatísticas: média e desvio padrão (média \pm DP), mediana e percentis 25 e 75, e foram analisados inferencialmente através dos testes estatísticos. Para a comparação entre os dois tipos de escova em cada grupo, foi utilizado o teste de Mann-Whitney, e entre os materiais utilizados na escovação para cada tipo de escova, foi utilizado o teste de Kruskal-Wallis. No caso de diferença significativa pelo teste de Kruskal-Wallis, foram utilizados testes de comparações múltiplas do referido teste. A escolha dos testes estatísticos foi devida ao número de amostras em cada combinação do material utilizado para escovação e tipo de escova ${ }^{15}$.

0 erro utilizado nas decisões dos testes estatísticos foi de $5 \%$. Os dados foram digitados na planilha Microsoft ${ }^{\circledR}$ EXCEL e o programa utilizado para obtenção dos cálculos estatísticos foi o IBM - SPSS versão $23^{15}$.

\section{RESULTADO}

Ao realizar a sobreposição das imagens da OCT, foi possível identificar as alterações de volume na superfície do esmalte, indicando a ocorrência ou não de desgaste em Pixels (Px).

Na Tabela 1 e na Figura 1, podemos observar as estatísticas do desgaste segundo o material utilizado e o tipo de escova. Desta Tabela, se destaca que as maiores diferenças entre os dois tipos de escovas utilizadas ocorreram nos grupos G2A e G2B, sendo o maior desgaste no grupo G2A $(16,09 \times 11,38)$. Nos grupos G1A e G1B, o que apresentou maior valor foi o grupo G1B $(13,20 \times 8,60)$, entretanto, para a margem de erro fixada (5\%), não houve diferenças significativas $(p>0,05)$ entre os dois tipos de escova em nenhum dos grupos. Em cada um dos tipos de escova, as médias menos elevadas ocorreram nos grupos G5A e G5B, com valores que 
variaram de 1,07 a 1,20. Quando foi utilizado a escova dental Curaprox ${ }^{\circledR}$, a média mais elevada ocorreu no grupo G2A $(16,09)$ e as demais médias variaram de 8,60 a 11,11; entre as amostras da escova Dental $\mathrm{K}^{\circledR}$, a média mais elevada ocorreu no grupo G1B $(13,20)$ e as demais médias dos grupos G2B a G4B variaram de 10,42 a 11,47.

Quando se consideram os resultados dos grupos levando em conta apenas o material utilizado para escovação (Tabela 1 e Figura 2), a média mais elevada ocorreu no grupo G2 $(13,73)$ e a média menos elevada foi no grupo G5 (1,13), com variação de 10,77 a 11,26. Foram registradas diferenças significativas $(\mathrm{p}<0,05)$ entre os grupos e, através dos testes de comparações múltiplas, se comprovam diferenças significativas entre o grupo G5 e cada um dos outros grupos avaliados.

Considerando o valor do desvio padrão em relação à média, se verifica uma variabilidade razoavelmente elevada nos grupos G2B, G4A, G1B e G1A, uma vez que os valores dos desvios padrão foram mais da metade das médias correspondentes. Nas demais situações, a menor variabilidade ocorreu no grupo G5A, com valor do desvio padrão inferior a um terço da média correspondente.

Tabela 1. Estatísticas do desgaste em Pixels (Px) segundo o material utilizado na escovação e o tipo de escova

\begin{tabular}{|c|c|c|c|c|}
\hline \multirow{5}{*}{ Grupo } & \multicolumn{2}{|c|}{ Tipo de escova utilizada } & \multirow{4}{*}{$\begin{array}{c}\begin{array}{c}\text { Independente da } \\
\text { escova }\end{array} \\
\text { Média } \pm \text { DP } \\
\text { Mediana (P25; P75) } \\
\end{array}$} & \multirow{5}{*}{ Valor de $p$} \\
\hline & $\begin{array}{l}\text { Curaprox }{ }^{\circledR} \text { Adulto } \\
\text { Ultra Macia }\end{array}$ & $\begin{array}{c}\text { Dental K }{ }^{\circledR} \text { Adulto } \\
\text { Macia }\end{array}$ & & \\
\hline & Média \pm DP & Média \pm DP & & \\
\hline & Mediana (P25; P75) & Mediana (P25; P75) & & \\
\hline & (GA) & (GB) & & \\
\hline \multirow[t]{2}{*}{ Grupo 1} & $8,60 \pm 4,33(A)$ & $13,20 \pm 7,55$ (A) & $10,90 \pm 6,29$ (A) & $p^{(1)}=0,347$ \\
\hline & $7,11(5,39 ; 12,56)$ & $12,89(7,22 ; 19,33)$ & $9,17(5,61 ; 13,86)$ & \\
\hline \multirow[t]{2}{*}{ Grupo 2} & $16,09 \pm 3,70$ (В) & $11,38 \pm 9,93(A)$ & $13,73 \pm 7,49$ (A) & $p^{(1)}=0,249$ \\
\hline & $18,11(12,11 ; 19,06)$ & $10,67(2,06 ; 21,06)$ & $14,56(8,58 ; 18,64)$ & \\
\hline \multirow[t]{2}{*}{ Grupo 3} & $11,11 \pm 5,27$ (АВ) & $10,42 \pm 5,10(A)$ & $10,77 \pm 4,90$ (А) & $p^{(1)}=0,917$ \\
\hline & $12,11(5,78 ; 15,94)$ & $10,00(6,44 ; 14,61)$ & $10,50(6,69 ; 15,75)$ & \\
\hline \multirow[t]{2}{*}{ Grupo 4} & $11,04 \pm 7,49(\mathrm{AB})$ & $11,47 \pm 5,15$ (A) & $11,26 \pm 6,06(A)$ & $p^{(1)}=0,675$ \\
\hline & $8,00(6,33 ; 17,28)$ & $11,67(6,72 ; 16,11)$ & $9,28(7,08 ; 14,67)$ & \\
\hline \multirow[t]{2}{*}{ Grupo 5} & $1,07 \pm 0,10(C)$ & $1,20 \pm 0,39$ (B) & $1,13 \pm 0,28(\mathrm{~A})$ & $p^{(1)}=0,906$ \\
\hline & $1,00(1,00 ; 1,17)$ & $1,00(1,00 ; 1,50)$ & $1,00(1,00 ; 1,14)$ & \\
\hline Valor de $\mathrm{p}$ & $\mathrm{p}^{(2)}=0,004^{*}$ & $\mathrm{p}^{(2)}=0,021^{*}$ & $\mathrm{p}^{(2)}<0,001^{*}$ & \\
\hline
\end{tabular}

*Diferença significativa no nível de 5,0\%. (1)Através do teste Mann-Whitney. (2)Através do teste Kruskal-Wallis com comparações do referido teste. Obs.: Se todas as letras entre parênteses são distintas, se comprovam diferenças significativas entre os grupos correspondentes. 


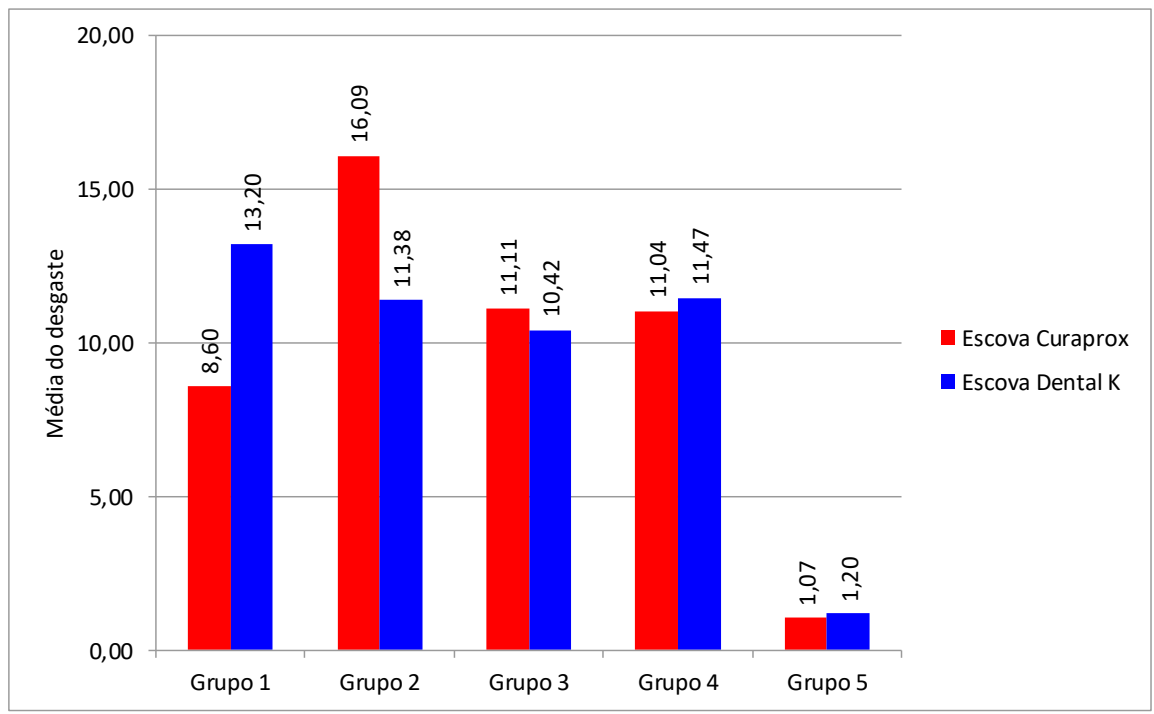

Figura 1. Média do desgaste em Pixels (Px) segundo o material utilizado para a escovação e o tipo de escova.

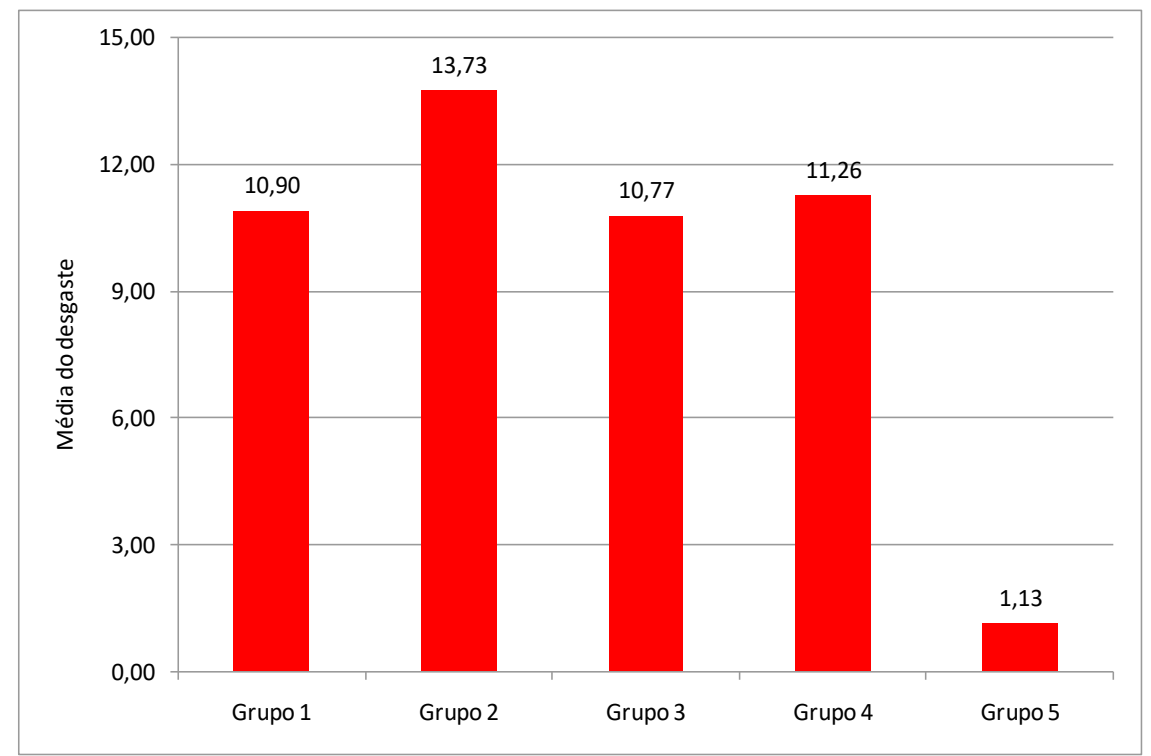

Figura 2. Média do desgaste em Pixels (Px) segundo o material utilizado na escovação.

\section{DISCUSSÃO}

Vários equipamentos são utilizados para observar a estrutura do esmalte dental, como Microscopia Eletrônica de Varredura (MEV), rugosimetria de superfície, processamento histológico, Microscopia Óptica, Tomografia por Coerência Óptica (OCT), entre outros ${ }^{13}$. Para analisar o esmalte dental no presente estudo, foi utilizada a OCT, um método de diagnóstico capaz de gerar imagens em tempo real e fornecer dados qualitativos e quantitativos ${ }^{16}$. Muitos trabalhos utilizaram a OCT como instrumento para leitura de estruturas dentais e mostraram a eficácia na visualização de alterações de superfície no esmalte dental, assim como na presente pesquisa ${ }^{17-19}$.

Dentes amarelados são queixas frequentes dos pacientes nos consultórios odontológicos e isso se deve ao aumento da valorização da estética dental e facial, como resultado do apelo da mídia por um sorriso saudável e cosmeticamente atraente. Objetivando satisfazer a necessidade dos 
consumidores, cresce no mercado a oferta dos chamados produtos de autocuidado (ou OTC, do inglês Over-The-Counter), cujo surgimento foi nos Estados Unidos da América, por volta dos anos 2000, como alternativa para tratar o manchamento dentário ${ }^{7}$.

Dentre as diversas apresentações comerciais dos produtos de autocuidado (enxaguantes bucais, tiras clareadoras, géis, dentre outros), os dentifrícios clareadores são amplamente divulgados e vendidos como cosméticos eficazes para obter clareamento dentário rápido e conveniente ${ }^{4,7}$. Os produtos cosméticos são aqueles que deverão ser esfregados, vertidos, polvilhados, introduzidos ou aplicados no corpo humano para a limpeza, o embelezamento e a promoção da atratividade ou a alteração da aparência, sendo, nesta categoriał, os materiais utilizados nesse estudo. Baseiam-se na tecnologia abrasiva para remoção de pigmentos extrínsecos, no entanto, geralmente não apresentam resultados tão satisfatórios quando comparados aos procedimentos clareadores executados por um profissional, além de poderem produzir efeitos nocivos aos dentes, como desgaste e sensibilidade dentária ${ }^{3}$.

De acordo com Rios et al. ${ }^{20}$, os dentifrícios, em geral, devem ser abrasivos o suficiente para realizar suas funções sem causar danos aos dentes, ou seja, os dentifrícios devem proporcionar máxima limpeza, com o mínimo de desgaste dos dentes. Diante de tal preocupação, é necessário que estudos sejam desenvolvidos para avaliar a abrasividade dos dentifrícios que estão disponíveis no mercado atualmente, porém a maioria dos cremes dentais não informa dados relacionados à quantidade de abrasivos nem à sua estrutura ${ }^{20}$.

Amaechi et al. ${ }^{21}$ analisaram dentifrícios que apresentavam como abrasivo a Sílica e outro com a Sílica Hidratada, e concluíram que o uso do dentifrício com Sílica resultou em uma maior abrasão quando comparado à Sílica Hidratada. Todavia, nesse trabalho, todos os dentifrícios utilizados apresentam a Sílica Hidratada como agente abrasivo em suas composições, com exceção do dentifrício Curaprox ${ }^{\circledR}$ Black is White, que possui Carvão Ativado associado ao referido componente (Quadro 1).

De acordo com Brooks et al. ${ }^{8}$, o Carvão Ativado é considerado um mineral abrasivo para os dentes e a incorporação destes em cremes dentais incita preocupações devido à escassez de evidências científicas que comprovem seus benefícios estéticos e ausência de cito-toxicidade, além da possibilidade de desgastes das estruturas dentárias, como comprovado no presente estudo, nos grupos G1A, G1B, G4A e G4B, que apresentaram um desgaste significativo da superfície do esmalte (Tabela 1, Figura 2). Observe-se que o uso de abrasivos que contêm Carvão ainda prevalece em algumas sociedades.

o Carvão Ativado inclui uma variedade de materiais ricos em carbono, amorfos ou altamente cristalinos, com alto grau de porosidade. Esse material geralmente é apolar, o que origina uma afinidade adsorvente por compostos orgânicos. Por esse motivo, o Carvão Ativado é utilizado principalmente na adsorção de compostos orgânicos, tanto no tratamento de efluentes como em outros processos, como purificação do ar, sistemas de recuperação de solventes e clarificação do açúcar, sendo bastante eficiente na remoção de poluentes ${ }^{22}$.

Com a divulgação desses produtos na internet, houve um aumento exponencial das vendas de dentifrícios clareadores e Carvão em pó ${ }^{8}$. A Curaprox ${ }^{\circledR}$ Black is White (G1A e G1B), que apresenta Carvão como principal agente ativo, é um exemplo de produto que teve seu lançamento acompanhado de extensas campanhas de publicidade, atentando-se para o fato de que este dentifrício pode custar até 20 vezes mais que uma pasta branqueadora convencional.

Para analisar os efeitos ocasionados pelos materiais abrasivos, neste trabalho, foi realizada uma escovação simulada na máquina MSET (Elquip ${ }^{\circledR}$ ), representando três anos de escovação, baseando-se na metodologia de outros estudos ${ }^{14}$. Contudo, foi possível observar que houve um maior desgaste nos Grupos 2A e 3A, provavelmente devido ao maior número de cerdas presentes

\footnotetext{
* Ferreira DG. Dentifrícios braqueadores: composição, efeitos, eficácia e cosméticos de venda livre em Portugal [dissertação]. Porto: Faculdade de Ciências da Saúde; 2017.
} 
na escova Curaprox ${ }^{\circledR}$ Adulto Ultra Macia do que na Dental $\mathrm{K}^{\circledR}$ - Adulto Macia, o que permitiu um maior contato das cerdas com o corpo de prova.

Alguns estudos ${ }^{4,5,7,23}$ que utilizaram dentifrício clareador da Colgate ${ }^{\circledR}$ constataram maior remoção de manchas quando comparado a outros dentifrícios clareadores empregados na pesquisa. Por meio deste trabalho, pode-se corroborar com tal resultado. 0 fato de o grupo G2 (Figura 2) ter provocado um maior desgaste na superfície do esmalte (90\%) deve-se provavelmente à concentração do abrasivo, ao tamanho ou à forma das partículas.

Há, no mercado, a comercialização exacerbada e sem controle de diversos tipos de produtos de autocuidado diferentes, cuja promessa é o clareamento dentário rápido e eficaz. Os dentifrícios clareadores são vendidos como cosméticos e utilizados pelos pacientes, na maioria dos casos, sem a supervisão de um profissional da área odontológica ${ }^{7}$. Existe uma ampla falta de clareza nas embalagens desses dentifrícios sobre a composição e a concentração de seus componentes, o que dificulta o conhecimento do consumidor acerca dos possíveis efeitos que o artigo que está sendo usufruído tem para a sua saúde.

Considerando que, devido à maior demanda e acessibilidade a esses produtos, estes podem ser utilizados pela população durante um longo período de tempo, levando ao desgaste intenso e prejudicial dos dentes. A perda acentuada da superfície do esmalte leva à sensibilidade e ao escurecimento dos dentes, à medida que a camada de esmalte se torna mais delgada. Logo, pode haver efeito contrário ao idealizado, quando esses produtos são usufruídos por tempo prolongado.

Apesar das limitações de um experimento in vitro, nosso trabalho mostrou que quando se comparou material e tipo de escova não houve diferenças estatísticas significativas, verificando-se que o maior desgaste ocorreu nos grupos G2A $(16,09)$ e G1B $(13,20)$. Quando se observam os resultados dos grupos, levando em consideração apenas o material utilizado, o maior desgaste ocorreu no grupo G2 $(13,73)$, sendo registradas diferenças significativas entre os grupos. Assim, é necessário que mais pesquisas sejam realizadas, especialmente clínicas.

\section{CONCLUSÃO}

Podemos concluir que:

- Todos os materiais utilizados, com exceção dos grupos controle (G5A e G5B), apresentaram desgaste significativo;

- $\mathrm{O}$ uso da escova Curaprox ${ }^{\circledR}$ Adulto Ultra Macia apresentou menor desgaste nos grupos G1A e G4A;

- 0 dentifrício Curaprox ${ }^{\circledR}$ Black is White, dos grupos G1B, obteve um desgaste semelhante ao Carvão Ativado (P.A.), dos grupos G4A e G4B;

- Os grupos G2B, G3A, G3B e G4B mostraram um desgaste semelhante;

- Os grupos que apresentaram maior desgaste foram aqueles que utilizaram o Colgate ${ }^{\circledR}$ Luminous White Instant para a escovação, com maior destaque no G2A, que utilizou a escova Curaprox ${ }^{\circledR}$ Adulto Ultra Macia.

\section{REFERÊNCIAS}

1. Schemehorn BR, Moore MH, Putt MS. Abrasion, polishing, and stain removal characteristics of various commercial dentifrices in vitro. J Clin Dent. 2011;22(1):11-8. PMid:21290981. 
2. Rosenthaler H, Randel H. Rotary reduction, enamel microabrasion, and dental bleaching for tooth color improvement. Compend Contin Educ Dent. 1998 Jan;19(1):62-7. PMid:9533352.

3. Lima FG, Rotta TA, Penso S, Meireles SS, Demarco FF. In vitro evaluation of the whitening effect of mouth rinses containing hydrogen peroxide. Braz Oral Res. 2012 May-Jun;26(3):269-74. http://dx.doi.org/10.1590/S1806-83242012000300014. PMid:22641448.

4. Horn BA, Bittencourt BF, Gomes OM, Farhat PA. Clinical evaluation of the whitening effect of over-thecounter dentifrices on vital teeth. Braz Dent J. 2014;25(3):203-6. http://dx.doi.org/10.1590/01036440201300053. PMid:25252254.

5. Bernardino RMP, Pedrosa MS, Silva AM, Silva BLC, Bezerra US, Moreno WG. Efetividade de dentifrícios clareadores sobre esmalte de dentes bovinos. Rev Salusvita. 2016;35(3):475-89.

6. Araújo DB, Silva LR, Campos EJ, Araújo RPC. In vitro study on tooth enamel lesions related to whitening dentifrice. Indian J Dent Res. 2011 Nov-Dec;22(6):770-6. http://dx.doi.org/10.4103/09709290.94665. PMid:22484868.

7. Roselino LMR, Tirapelli C, Pires-de-Souza FC. Randomized clinical study of alterations in the color and surface roughness of dental enamel brushed with whitening toothpaste. J Esthet Restor Dent. 2018 Sep;30(5):383-9. http://dx.doi.org/10.1111/jerd.12379. PMid:29603865.

8. Brooks JK, Bashirelahi N, Reynolds MA. Charcoal and charcoal-based dentifrices: a literature review. J Am Dent Assoc. 2017 Sep;148(9):661-70. http://dx.doi.org/10.1016/j.adaj.2017.05.001. PMid:28599961.

9. Tawakoli PN, Becker K, Attin T. Abrasive effects of diamond dentifrices on dentine and enamel. Swiss Dent J. 2018 Jan;128(1):14-9. PMid:29533050.

10. International Organization for Standardization - ISO. ISO TR 11405: dental materials: guidance on testing of adhesion to tooth structure. Switzerland: ISO; 2015.

11. Menezes MM, Firoozmand LM, Huhtala MFR. Avaliação do desgaste superficial do esmalte escovado com dentifrícios e submetido à ação de agentes branqueadores. Cienc Odontol Brasil. 2003 JanMar;6(1):44-50. http://dx.doi.org/10.14295/bds.2003.v6i1.190.

12. International Organization for Standardization - ISO. ISO TR 14569-1: dental materials - guidance on testing of wear resistance. Part 1: wear by tooth brushing. Switzerland: ISO; 2007.

13. Tostes NE, Baptista NB, Carvalho OB Jr, Franciscone PAS, Pires HC. Avaliação de desgaste produzidos em esmalte por creme dentais clareadores. Rev Odontol Araçatuba. 2009 Jul-Dez;30(2):9-13.

14. Monteiro B, Spohr AM. Surface roughness of composite resins after simulated toothbrushing with different dentifrices. J Int Oral Health. 2015 Jul;7(7):1-5. PMid:26229362.

15. Altman DG. Practical statistics for medical research. London: Chapman \& Hall; 1991.

16. Mota CC, Fernandes LO, Cimões R, Gomes AS. Non-invasive periodontal probing through fourierdomain optical coherence tomography. J Periodontol. 2015 Sep;86(9):1087-94. http://dx.doi.org/10.1902/jop.2015.150047. PMid:25879790.

17. Mandurah MM, Sadr A, Shimada Y, Kitasako Y, Nakashima S, Bakhsh TA, et al. Monitoring remineralization of enamel subsurface lesions by optical coherence tomography. J Biomed Opt. 2013 Apr;18(4):046006. http://dx.doi.org/10.1117/1.JB0.18.4.046006. PMid:23563920.

18. Min JH, Inaba D, Kwon HK, Chung JH, Kim BI. Evaluation of penetration effect of resin infiltrant using optical coherence tomography. J Dent. 2015 Jun;43(6):720-5. http://dx.doi.org/10.1016/j.jdent.2015.03.006. PMid:25862274.

19. Marcauteanu C, Bradu A, Sinescu C, Topala FI, Negrutiu ML, Podoleanu AG. Quantitative evaluation of dental abfraction and attrition using a sweptsource optical coherence tomography system. J Biomed Opt. 2014 Feb;19(2):21108. http://dx.doi.org/10.1117/1.JBO.19.2.021108. PMid:24042447. 
20. Rios ACF, Lopes SCFL, Dantas TS, Oliveira VMB, Santos LB. Abrasivos: uma análise de dentifrícios comercializados em Salvador. Revista Bahiana de Odontologia. 2014 Dez;5(3):141-52.

21. Amaechi BT, Higham SM, Podoleanu AG, Rogers JA, Jackson DA. Use of optical coherence tomography for assessment of dental caries: quantitative procedure. J Oral Rehabil. 2001 Dec;28(12):1092-3. http://dx.doi.org/10.1046/j.1365-2842.2001.00840.x. PMid:11874506.

22. Brooks JK, Bashirelahi N, Reynolds MA. More on charcoal and charcoal-based dentifrices. J Am Dent Assoc. 2017 Nov;148(11):785. http://dx.doi.org/10.1016/j.adaj.2017.09.027. PMid:29080603.

23. Silva MFR, Tomo S, Oliveira CV, Alves DP, Lima DP, Pires HC. Avaliação in vitro da eficácia de dentifrícios de ação clareadora. Arch Health Invest. 2015;4(2):35-9.

\section{CONFLITOS DE INTERESSE}

Os autores declaram não haver conflitos de interesse.

\section{*AUTOR PARA CORRESPONDÊNCIA}

Letícia Santos Alves de Melo, UFPE - Universidade Federal de Pernambuco, Centro de Ciências da Saúde, Departamento de Prótese e Cirurgia Buco Facial, Av. Prof. Moraes Rego, 1235, Cidade Universitária, 50670-901 Recife - PE, Brasil, e-mail: leticia.melo41@gmail.com

Recebido: Julho 31, 2019

Aprovado: Setembro 18, 2019 